\title{
A case of "voluntary nystagmus" and head tremor
}

\author{
John Lee, Michael Gresty
}

\begin{abstract}
A 24-year-old woman presented with nystagmus and head tremor. Both were transient and could be executed simultaneously at different frequencies of oscillation. It is unusual for these two movements, which can be voluntary, to occur together. Suspicions of an acquired disease were not confirmed on further investigation using DC-coupled electro-oculography and angular accelerometry, and on an admission by the patient that the nystagmus could be voluntary. Voluntary nystagmus can become semi-automatic and a patient may be able to simulate more than one "involuntary movement" simultaneously yet at different frequencies.
\end{abstract}

(F Neurol Neurosurg Psychiatry 1993;56:1321-1322)

Voluntary nystagmus consists of oscillations of the eyes which can be produced partly "at will" by (some) normal subjects and is occasionally encountered in patients with functional disturbances. On analysis they are sinusoidal in trajectory with peak velocities which approach those of the main sequence for saccades. ${ }^{1}$ Some voluntary nystagmus involves eye movements which are similar to ocular flutter which is a sign of brainstem disease. Flutter is also composed of "back-toback" saccades and may thus present a minor problem of differential diagnosis.

We report an unusual case of voluntary nystagmus accompanied by head tremor, which was also voluntary in nature, but which presents a more difficult differential diagnosis.

Moorfields Eye

Hospital, London, UK

J Lee

MRC Human

Movement and

Balance Unit,

National Hospital for

Neurology and

Neurosurgery, Queen

Square, London

WC1N 3BG, UK

M Gresty

Correspondence to:

M Gresty

Received 20 July 1992.

Accepted 25 September 1992 jugate horizontal oscillations which were provoked particularly by vergence and pursuit movements. About the same time her head executed an irregular "no-no" tremor. On repeated provocation the duration of the bursts of nystagmus and tremor tended to diminish and she would eventually break off the confrontation by grimacing. Neither the nystagmus nor head tremor were observed during surveillance which was carried out unknown to the patient.

General systems and a full ophthalmological examination revealed no other abnormalities. In particular there was no nuchal rigidity to suggest dystonia.

During supportive discussion the patient eventually admitted that she had been able to wobble her eyes since being a teenager. She said that recently it seemed to have become out of her control; particularly when being examined by a doctor. We also discovered later that she had been investigated as an inpatient some years before for a "functional" bowel disorder.

\section{Head and eye movement recordings}

Horizontal eye movements were recorded with DC-coupled electro-oculography and head movement in the coronal plane was transduced by an angular accelerometer mounted on the skull with a head band.

The eye movements always took the same form of sinusoidal oscillations at a frequency of $15 \mathrm{~Hz}$ with amplitudes up to $30^{\circ}$ from left or right through primary gaze with peak velocities up to $500 \mathrm{o} / \mathrm{s}$. Velocities of normal saccades or fast phases of vestibular nystagmus were comparable and all were consistent with established norms. ${ }^{2}$ Head tremor was irregular in waveform consisting of jerks and sinusoidal oscillations and with peak acceleration occasionally up to $2000 \mathrm{o} / \mathrm{s}^{2}$ but more typically of the order of $800 \mathrm{o} / \mathrm{s}^{2}$, which would be typical of normal head shaking to indicate "no-no". Corresponding amplitudes of head movement were up to circa $\pm 12^{\circ}$ (fig A, B). The patient's head movements were reminiscent of some tremors of the head accompanied by dystonia. ${ }^{3}$ They could be simulated by attempting to tense one's neck muscles and shuddering.

Nystagmus and head tremor could occur independently and the head movement could 
A

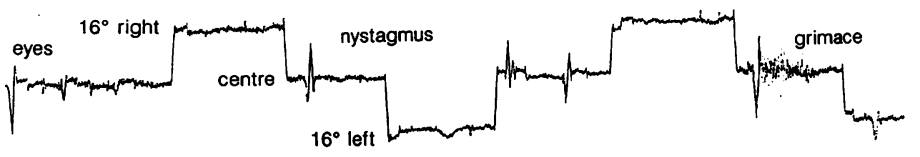

head acceleration

B

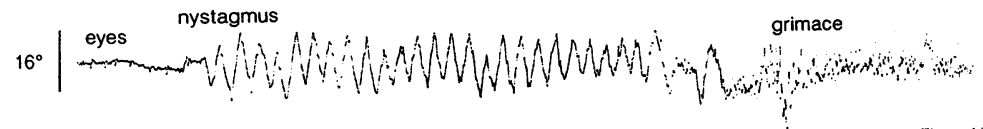

500\%s/s (nead
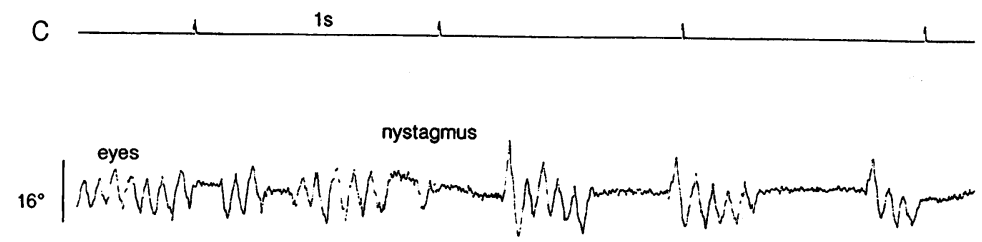

500\%/s head acceleration

Figure A) Conjugate eye movements in the horizontal plane recorded as a "cyclopean" eye. Calibration saccades showing intermittent bursts of voluntary nystagmus;

B) Recordings of horizontal eye displacement and head acceleration in the coronal plane showing: the quasi-sinusoidal oscillatory structure of nystagmus, the irregular structure of the head movement with unidirectional jerks and oscillations indicated, the temporal independence of relative onset and offset of the nystagmus and head shaking; C) Recordings of horizontal eye displacement and head acceleration in the coronal plane showing bursts of nystagmus progressively diminishing in duration as the patient was challenged with a provocative manoeuvre. eyes is not characteristic of any commonly encountered neurological disorder. However, the unusual presentation of head tremor with a nystagmus, which in isolation, might be diagnosed as voluntary, raised the level of our suspicions of acquired disease.

The fatigueability of voluntary nystagmus is almost pathognomonic. ${ }^{4}$ This is not the case with movement disorders of the head and limbs of functional origin which may be sustained indefinitely, ${ }^{5}$ particularly if they are rhythmical at a frequency characteristic of natural voluntary or involuntary motion. Accordingly, the differential diagnosis between "essential" head tremor and head movement of functional origin can be difficult. Diagnosis of the head movement disorder in this patient was facilitated by the concurrence of nystagmus which was admittedly voluntary in origin.

A significant feature of the abnormal movements in this patient was that they were executed simultaneously at different frequencies of oscillation which is not easy to perform voluntarily; for example, walking whilst attempting to swing the arms out of synchrony is difficult. A frequent ploy to reveal the nature of a head or arm tremor which is suspected to be simulated is to ask the patient to execute movements of some other limb in time to an externally imposed tempo of different frequency; in most cases the task and tremor cannot be executed concurrently and the performance of one or other suffers.

In common with somatic movement disorders of functional origin one should be aware that voluntary nystagmus can become semiautomatic and that a patient may be able to simulate "involuntary movements" simultaneously at different frequencies in different parts of the body.

be sustained longer than the nystagmus. When eye and head movements concurred their onsets or offsets were not necessarily synchronous. During sustained provocation a train of nystagmus oscillations tended to disintegrate into bursts with diminishing numbers of cycles per burst (fig $\mathrm{C}$ ).

\section{Discussion}

The phenomenon of a transient head tremor coupled with flutter-like oscillations of the

1 Shults WT, Stark L, Hoyt WF, Ochs AL. Normal saccadic structure of voluntary nystagmus. Arch Ophthalmol 1977;95:1399-404.

2 Collewijn H, Erkelens CJ, Steinman RM. Binocular coordination of human horizontal saccadic eye movements. f Physiol 1988;404:157-82.

3 Findley LJ, Gresty MA. Head, facial and voice tremor In: Jankovic J, Tolosa $\mathrm{E}$, eds. Advances in neurology, vol 48. Facial Dyskinesias. New York: Raven, 1988:239-53.

$4 \mathrm{Zahn} \mathrm{JR}$. Incidence and characteristics of voluntary nystagmus. F Neurol Neurosurg Psychiatry 1978;41:617-23.

5 Fahn S. Atypical, rare and unclassified tremors. In Findley LJ, Capildeo R (eds) Movement Disorders. Tremor. London: Macmillan 1984:431-43. 